\title{
Thermo-dynamic plasma expansion acceleration in asymmetric spontaneous fast magnetic reconnection
}

\author{
Tohru Shimizu and Masayuki Ugai \\ Department of computer science, Ehime university, Bunkyo town, Matsuyama, Ehime 790-8577, Japan
}

(Received June 21, 2000; Revised November 20, 2000; Accepted January 5, 2001)

\begin{abstract}
Asymmetric spontaneous fast magnetic reconnection model, in which the current sheet is initially put between two antiparallel straight magnetic field regions with different magnetic intensities, is studied by two-dimensional magnetohydrodynamic (MHD) simulations. Especially, the supersonic expansion acceleration process which has been already observed in the symmetric case is focused on. In this paper, we find that if the initial asymmetry features are weak and one side of the magnetic field regions consists of sufficiently low beta plasma, the supersonic expansion acceleration process is generated in the reconnection jet region, like the symmetric case. Hence, in such an asymmetric case, a fast shock is formed at the top of the magnetic loop.
\end{abstract}

\section{Introduction}

The spontaneous fast magnetic reconnection model proposed by Ugai and Tsuda (1977) describes a new type nonlinear instability that results from the positive feedback between macroscopic reconnection flows and microscopic anomalous resistivities. In this model, as the magnetic diffusion region is spontaneously localized by the nonlinear instability, slow shocks are generated, so that an active reconnection process is established. Then, as the reconnection process proceeds, a magnetic loop (plasmoid) is formed in the downstream of the fast plasma jet. In contrast to the Petschek model, the spontaneous fast magnetic reconnection model can have steady plasma jets exceeding the Alfven velocity measured in the upstream field region of the reconnection layer. In fact, according to our two-dimensional magnetohydrodynamic (MHD) simulations, the final velocity of the plasma jet is observed to be superfast and reach 1.4 times of the Alfven velocity, which is maintained until the jet encounters a fast shock generated in front of the plasmoid.

In recent studies, we found theoretically that the plasma acceleration is caused by the thermo-dynamic supersonic expansion acceleration mechanism (Shimizu and Ugai, 2000), which is caused, as follows. In the first step, the plasma is accelerated upto the Alfven velocity measured in the upstream field region by one pair of slow shocks associated with the fast reconnection process. At the time, the Alfven velocity in the upstream field region can exceed the local sound velocity in the downstream jet region, so that the plasma jet generated by slow shocks can be supersonic. In the second step, the supersonic plasma jet expands in the direction normal to the jet, associated with the swelling of the magnetic loop. The expansion of the plasma jet causes the thermodynamic supersonic plasma expansion acceleration, leading

Copy right (C) The Society of Geomagnetism and Earth, Planetary and Space Sciences (SGEPSS); The Seismological Society of Japan; The Volcanological Society of Japan; The Geodetic Society of Japan; The Japanese Society for Planetary Sciences. to the plasma acceleration beyond the Alfven velocity. Finally, the plasma jet terminates at a fast shock in front of the magnetic loop, leading to the enhancement of the swelling of the magnetic loop.

In this paper, we mainly study an asymmetric field case, in which two magnetic field regions separated by the current sheet have different field intensities and the same (uniform) plasma density. In Section 2, the simulation procedures are mentioned, and in Section 3, the results are shown. Sections 3 and 4 are devoted to the discussions and summary.

\section{Procedures of MHD Simulation}

A two-dimensional MHD simulation is executed in an initial asymmetric antiparallel magnetic field region, in which the initial magnetic field intensity $B_{x 2}$ in the lower side magnetic field region is higher than $B_{x 1}$ in the upper side magnetic field region, as shown in Fig. 1. In this paper, the value $B_{x 2}$ is taken to be 1.5 (or 1.0 for the symmetric case), and $B_{x 1}$ is fixed to be 1.0. The plasma density is initially uniform $\left(\rho_{0}=1\right)$. The initial pressure $P_{0}$ is determined by the pressure balance equation $P_{0}+B_{x 0}^{2}=C ; C$ is constant in space and given by $P_{2}+B_{x 2}^{2}\left(P_{2}\right.$ is the initial pressure in the lower side magnetic field region and set to be 0.1 ). The so-called symmetry boundary condition, which works as a wall boundary condition, is embedded in the left side, upper and lower boundary lines in Fig. 1. Free (open) boundary condition is embedded in the right side boundary line.

The details of the simulation procedures are mentioned in our previous papers (Ugai and Shimizu, 1996; Shimizu and Ugai, 2000). The normalization is as follows. The distances $\vec{r}=(x, y)$ are normalized by $d_{0}$, which is the half width of the initial current sheet. The magnetic field $\vec{B}$ is by $B_{x 1}$ in the upper field region, pressure $P$ is by $B_{x 1}^{2} / \mu_{0}$, density $\rho$ is by $\rho_{0}$, current $J_{z}$ is by $B_{x 1} / \mu_{0} d_{0}$, plasma velocity $\vec{u}$ is by $V_{A 1}=B_{x 1} / \sqrt{\mu_{0} \rho_{0}}$ and time is by $d_{0} / V_{A 1}$. The anomalous resistivity is assumed to be $\eta=k_{R}\left[V_{D}(\vec{r}, t)-V_{C}\right]$ for $V_{D}>$ $V_{C}$ and $\eta=0$ for $V_{D}<V_{C}$, and assumed to be isotropic. 


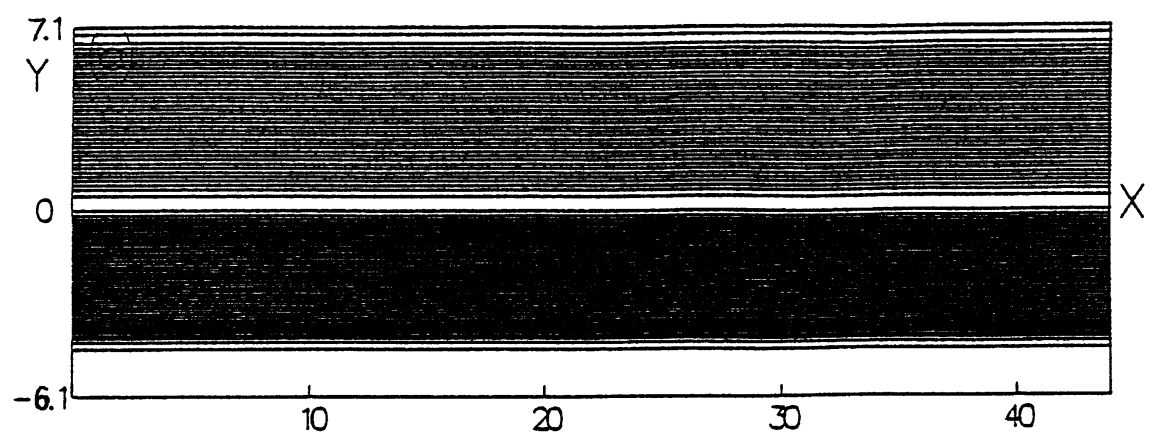

Fig. 1. Initial magnetic field. The upper and lower regions have $B_{x 1}=1.0$, and $B_{x 2}=1.5$, respectively.
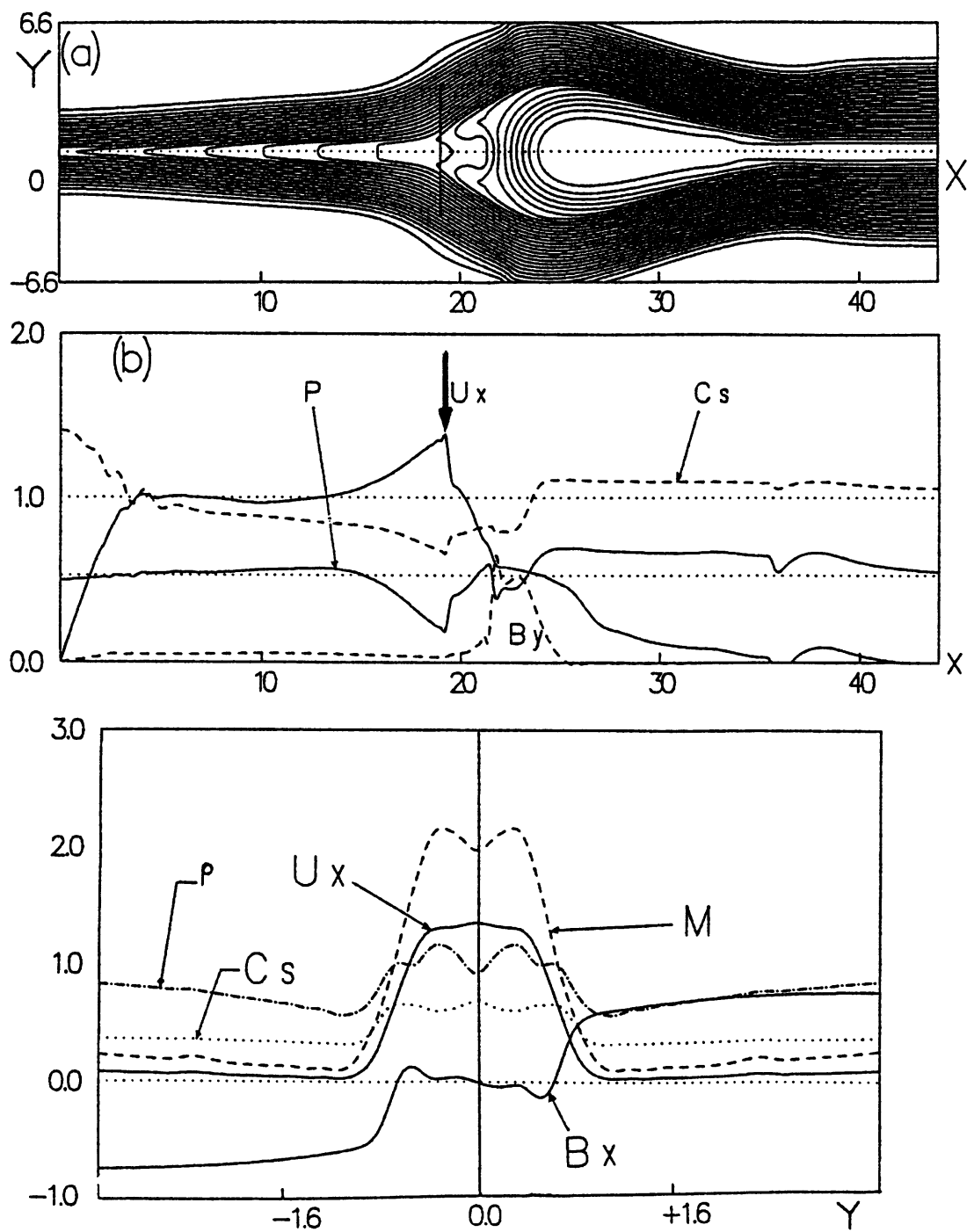

Fig. 2. Symmetric case. (a) Magnetic field at the time when the reconnection process has been fully developed. (b) The profiles of the pressure $P$, velocity $U_{x}$, magnetic field $B_{y}$ and local sound velocity $C_{s}$ along the $x$-axis. A downward thick arrow indicates the position of the fast shock. (c) The profiles of $\rho, C_{s}, B_{x}, U_{x}$ and $M=U_{x} / C_{s}$ along the $y$-directional line at $x=19.0$, which is including the max velocity $U_{x}$ point.

The value $V_{D}$ is given by $|\vec{J} / \rho|, k_{R}=0.002$ and $V_{C}=4.0$. The anomalous resistivity is normalized by $\mu_{0} d_{0} V_{A 1}$. The simulation box is taken to be $L_{x}=44.0$ and $L_{y}=13.2$.

Then, the outline of the simulation is as follows. In time $0<t<4$, the magnetic field configuration around the origin is destabilized by the small resistive disturbance. After the resistive disturbance is removed at time $t=4$, an Xtype field configuration formed locally at the origin causes the current sheet thinning, due to the resulting weak plasma flows. The thinning of the current sheet eventually enhances 

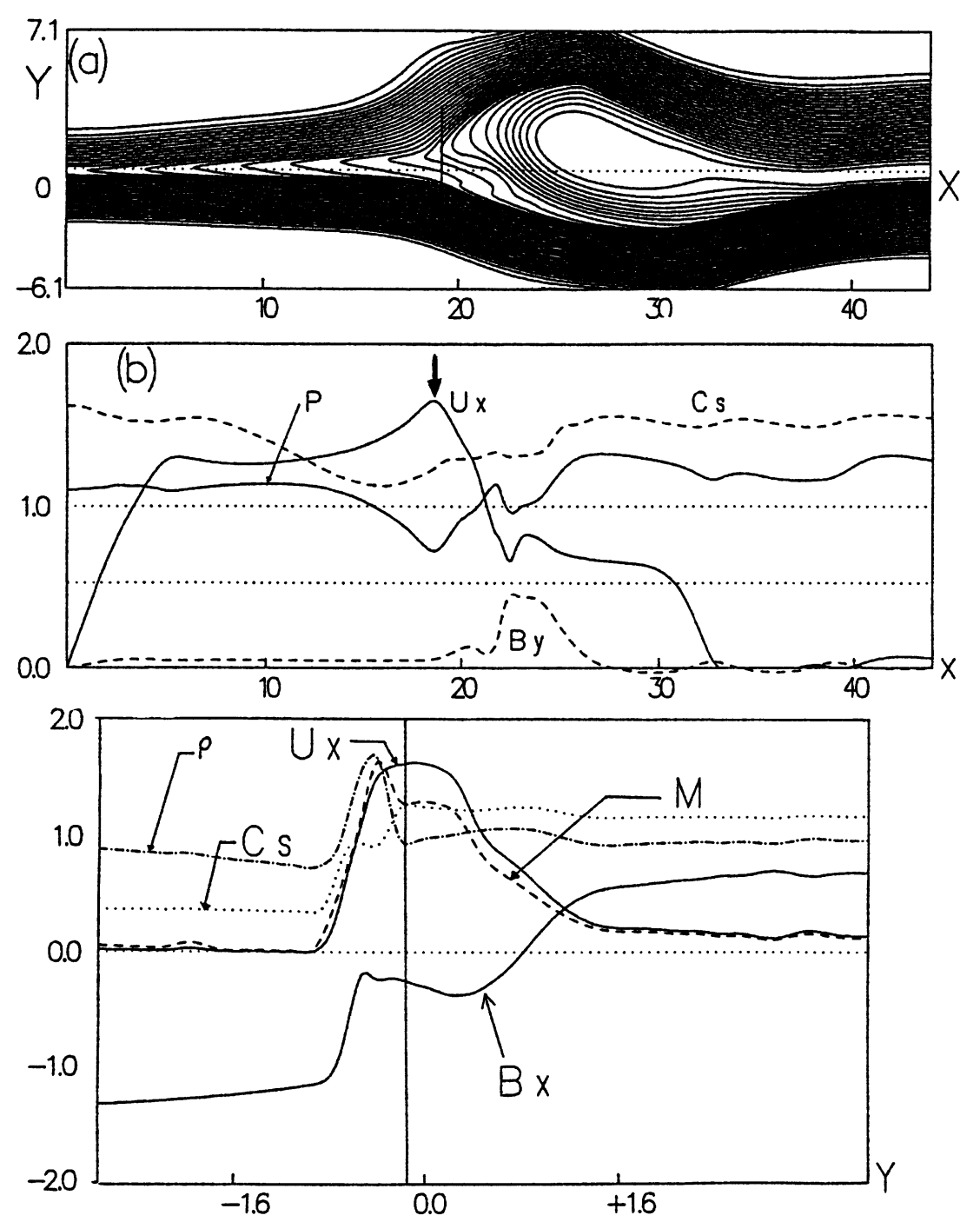

Fig. 3. Asymmetric case $\left(B_{x 1}=1.0\right.$, and $\left.B_{x 2}=1.5\right)$. In (b), the profiles are obtained along a horizontal line $(y=0.17)$ including the max velocity point. The other caption is similar to Fig. 2. (c) This is also similar to that of Fig. 2. But, it is plotted along a $y$-directional line $(x=18.6)$.

the current-driven anomalous resistivity around the origin. Since the anomalous resistivity and magnetic reconnection flow simultaneously grow to enhance each other, the fast magnetic reconnection mechanism is spontaneously built up $(t>30)$.

\section{Simulation Results}

\subsection{Symmetric case}

Figure 2 is obtained in the symmetric case $\left(B_{x 1}=B_{x 2}=\right.$ 1.0). Figures 2(a) and (b) respectively show the magnetic field and profiles of the pressure $P$, plasma jet's velocity $U_{x}$, y-directional field component $B_{y}$ and local sound velocity $C_{s}$ along the $x$-axis at the time when the fast reconnection has been fully developed. In Fig. 2(a), a growing magnetic loop associated with the reconnection process is running in the positive $x$ direction. The diffusion region is strongly localized around the origin $(x<3)$ and the region size is much smaller than the simulation box $(0<x<44)$, so that the Petschek reconnection model is well established in $0<x<13$ in Figs. 2(a) and (b). In fact, $U_{x}$ in $4<x<13$ almost coincides with the Alfven velocity $V_{A 1}=V_{A 2}=1.0$ measured in the initial upstream field region. By the way, the region of $4<x<13$ is supersonic, because of $U_{x}>C_{s}$. Considering that the local sound velocity $C_{s}$ is almost equal to the local fast wave velocity in the high beta plasma jet, it means that the plasma jet generated by slow shocks can be superfast. However, in $13<x<19$, we can easily find a region in which $U_{x}$ largely exceeds the Alfven velocity. Since the field intensity and Alfven velocity in the upstream field region gradually decreases, as the reconnection proceeds, such a high-speed plasma jet cannot be explained by the slow shock acceleration associated with the fast reconnection process. Instead, it was found that the supersonic expansion acceleration mechanism causes the acceleration, as mentioned in the introduction. Since this simulation is started from the symmetric magnetic field, everything proceeds symmetrically around the $x$-axis. Figure 2(c) shows the profiles of the plasma density $\rho, B_{x}, U_{x}$ and Mach number $M=U_{x} / C_{s}$ along the $y$-directional line at $x=19.0$. As shown in Fig. 2(c), the superfast jet region $(M>1)$ symmetrically spreads in the whole of the reconnection jet located between two slow shocks $(y=0.8$ and -0.8$)$. These slow 
shocks are respectively combined with the transient intermediate waves (Ugai and Shimizu, 1994).

\subsection{Asymmetric case}

Figure 3 shows the result obtained in an asymmetric case for $B_{x 1}=1.0$ and $B_{x 2}=1.5$. Figures 3(a), (b) and (c) show the magnetic field and profiles similar to those of Figs. 2(b) and (c), respectively. Note that the profiles in Fig. 3(b) and (c) are respectively plotted along the horizontal line $(y=0.17)$ and vertical line $(x=18.6)$ which include the point of the fastest jet velocity $U_{x}$ in the entire simulation region. The generated magnetic loop is distorted, due to the asymmetric initial field configuration. Especially, the magnetic loop is largely swelling toward the upper side field region, which has a weaker field intensity than the lower side field region. Also in this case, a superfast jet region $\left(U_{x}>C_{s}\right)$ can be seen in Figs. 3(b) and (c). However, in Fig. 3(b), the superfast (supersonic) region seems to be narrower than the symmetric case (Fig. 2(b)). In addition, Fig. 3(c) shows that the maximum of the Mach number $(=1.7)$ is lower than that $(=2.2)$ of the symmetric case, and the peak of the Mach number shifts to the side of the high intensity field region (left side; $y<0$ ). Note that the $U_{x}$ in Fig. 3(c) is almost uniform in the jet region (roughly, $-0.5<y<0.5$ ). It suggests that the reconnection jet almost reaches a steady state.

\section{Discussions}

\subsection{Generation of the superfast jet}

Basically, the supersonic expansion acceleration mechanism requires that the reconnection jet generated by slow shocks is superfast. Now, the Alfven velocities in the upper and lower side magnetic field regions are initially $V_{A 1}=$ $B_{x 1} / \sqrt{\rho_{1}}$ and $V_{A 2}=B_{x 2} / \sqrt{\rho_{2}}$, respectively. The Alfven velocity may be considered to be the maximum jet velocity generated by the slow shock, which is just a switch off shock. In the symmetric case, in fact, the reconnection jet's velocity becomes close to the Alfven velocity $\left(V_{A 1}=V_{A 2}\right)$, as shown in Fig. 2(b). But, in the asymmetric case started from the uniform plasma density assumed here, the slow shock is always weaker than the switch off shock. In addition, even in a steady state of the asymmetric reconnection jet, the velocity $U_{x}$ is almost uniform in the whole of the jet region, as shown in Fig. 3(c). It suggests that the plasma acceleration by the lower side strong slow shock $(y<0)$ is prevented by the slow jet generated by the upper side weak slow shock $(0<y)$, and finally, the reconnection jet velocity must have a constant velocity less than $V_{A 2}$. On the other hand, because of the uniform density and pressure balance $P+B^{2}=$ const. assumed initially, the sound velocity $C_{s}=\sqrt{P / \rho}$ in the upper side field region $(0<y)$ is higher than that of the lower side field region $(y<0)$. Accordingly, also in the reconnection jet region, the sound velocity in the upper side of the jet tends to be higher than the sound velocity in the lower side.

Hence, because of the uniform jet velocity and non-uni- form sound velocity in the upper and lower side of the jet region, the upper side region of the reconnection jet tends to have lower Mach number than the lower side of the jet region. As a results, as shown in Fig. 3(c), the higher Mach number jet region tends to shift to the lower side jet region in which the plasma is supplied from the strong intensity field region $(y<0)$.

\subsection{Expansion of the superfast jet}

The swelling of the magnetic loop (plasmoid) causes the expansion of the plasma jet in the direction normal to the jet, due to the magnetic field tension. Since the swelling is intrinsically caused by the break of the initial pressure balance between the magnetic loop and surrounding magnetic field region in the fast reconnection process, the expansion of the reconnection jet should always occur in front of the magnetic loop. Hence, once the supersonic jet is generated by slow shocks, the supersonic jet is accelerated by the supersonic expansion acceleration mechanism.

\section{Summary}

Asymmetric spontaneous fast reconnection process was studied in MHD simulation of initial uniform plasma density. We found that when one of the two antiparallel magnetic field regions separated by the current sheet is filled with sufficiently low beta plasma and the two field intensities $B_{x 1}$ and $B_{x 2}$ is close (asymmetric effect is weak), the supersonic adiabatic expansion acceleration process can be caused, like the symmetric case. However, the obtained result suggests that as the field intensities in the upper and lower magnetic field regions are largely different, the acceleration becomes weaker and may finally not work. In the next step, the supersonic expansion acceleration process must be theoretically studied in various asymmetric cases which have various plasma conditions. It may be partially related to the general works for asymmetric reconnection models done by Semenov et al. (1983).

\section{References}

Semenov, V. S., I. V. Kubyshkin, M. F. Heyn, and H. K. Biernat, Field-line reconnexion in the two-dimensional asymmetric case, J. Plasma Phys., 30, 321-344, 1983.

Shimizu, T. and M. Ugai, Adiabatic expansion acceleration mechanism of superfast jets in the spontaneous fast magnetic reconnection model, Phys. Plasmas, 7(6), 2417-2424, 2000.

Ugai, M. and T. Tsuda, Magnetic field line reconnection by localized enhancement of resistivity. Part 1. Evolution in a compressible MHD fluid, J. Plasma Phys., 17(P3), 337-356, 1977.

Ugai, M. and T. Shimizu, Computer Studies on Noncoplanar Slow and Intermediate Shocks Associated with the Sheared Fast Reconnection Mechanism, Phys. Fluids B, 1(2), 296-307, 1994.

Ugai, M. and T. Shimizu, Computer studies on the spontaneous fast reconnection mechanism in three dimensions, Phys. Plasmas, 3, 853-862, 1996

T. Shimizu (e-mail: shimizu@cs.ehime-u.ac.jp) and M. Ugai 